\title{
Some Notes on the Distribution of Mersenne Primes
}

\author{
Sibao Zhang ${ }^{1}$, Xiaocheng Ma ${ }^{1}$, Lihang Zhou ${ }^{2}$ \\ ${ }^{1}$ Department of Mathematics, Kashgar Teachers College, Kashgar, China \\ ${ }^{2}$ Department of Computer Science, Guangdong Technical College of Water Resources and Electric Engineering, \\ Guangzhou, China \\ E-mail: sibao98@sina.com
}

Received July 13, 2010; revised August 19, 2010; accepted August 21, 2010

\begin{abstract}
Mersenne primes are a special kind of primes, which are always an important content in number theory. The study of Mersenne primes becomes one of hot topics of the nowadays science. It has not settled that whether there exist infinite Mersenne primes. And several of conjectures on the distribution of it provided by scholars. Starting from the Mersenne primes known about, in this paper we study the distribution of Mersenne primes and argued against some suppositions by data analyzing.
\end{abstract}

Keywords: Mersenne Primes, Distribution, Zhou Conjecture, Number Theory

\section{Introduction}

In 300 B.C , Euclid, famed ancient Greek mathematician proved that there are infinitude prime numbers by contradiction, and raised that a small amount of prime numbers could be expressed in the formulation of $2^{p}-1(p$ is a prime number). After that, many famous mathematicians including mathematical masters like Fermat, Descartes, Leibniz, Goldbach, Euler, Gauss all researched into the prime numbers of this kinds of special formulation. But M. Mersenne, the French mathematician in 17th century and founder of Institute of France, was the first one to research into the prime number of $2^{p}-1$ formulation deeply and systematically. These kinds of formulation like $2^{p}-1$ is named as "Mersenne number" and expressed as $M_{p}$ to commemorate him. If a Mersenne number is a prime number, then is named as "Mersenne prime". The Mersenne prime seems like very simple, but the calculation of it is very complex. As the increase of index $p$, the calculation increases more complex. It not only need advanced theory and practiced skills, but arduous calculation is needed to validate whether a Mersenne number is prime or not. People have only discovered 47 Mersenne primes in the past 2300 years [1].

It should be noted that we have only settled the sequence of first 39 primes, while the last 8 primes left. That is, there is no other prime number $p$ which makes $2^{p}-1$ to be a prime number in the range of $2 \leq p \leq 13$ 466 917. And we still cant assure that whether exists other prime number $p$ which makes $2^{p}-1$ to be prime number in the range of $20996011 \leq p \leq 43112609$. Though we have not discovered other prime $p$ which makes $2^{p}-1$ to be a prime number by checked the range at least once, but twice could confirm the seating arrangement [2].

It is not known whether the set of Mersenne primes is infinite. But researching on the attribution of Mersenne Primes is very important for the seeking of new Mersenne primes and exploring whether exist infinitude Mersenne primes. From the known Mersenne primes, the distribution of these special kinds of prime number is either sparse or dense in the positive integer, so very anomalous. In the long-term exploring, mathematicians have advanced some kind of suppositions. For example, mathematicians like Shanks from England, Bertrand from France, Ramanujan from India, Gilles from America and Brillhart from Germany have all supposed the distribution of Mersenne primes. The common point of their supposition is that they all presented as asymptotic expression.

In 1992, Haizhong Zhou [3], famed Chinese mathematician and linguist advanced the precise formulation of the Mersenne prime distribution: If $2^{2^{n}}<p<2^{2^{n+1}}(n=$ $0,1,2,3, \ldots)$, then the amount of Mersenne primes is $2^{n+1}-1$. The accurate and beautiful expression is made by him. Zhou conjecture has not been proved or disproved, and becomes a well-known mathematical problem [4].

Some researchers have raised the suppositions for the distribution of Mersenne primes. In this article we would 
argue against the deduction and raise different views by data analyzing for several suppositions.

\section{Questions and View Points}

\subsection{Questions}

In 1995, based on Zhou conjecture, Suwen Chen tried to make a further discussion on the distribution of Mersenne primes [5]. In the paper he defined that: Sequence $\boldsymbol{R}$ consists of primes $p$ which makes $2^{p}-1$ to be prime and numbers like $2^{2^{k}} \quad(k=0,1,2, \ldots)$ in ascending, and the item No. $n$ marked $\boldsymbol{R}(n) ; \boldsymbol{P}$ is the sequence consists of primes $p$ which makes $2^{p}-1$ primes in ascending, while the item No. n marked $\boldsymbol{P}(n) ; \boldsymbol{Q}$ is the sequence consists of numbers like $\boldsymbol{Q}(n)=2^{2^{n}} \quad(n=0,1,2,3, \ldots)$. By analyzing the first 35 items settled by $\boldsymbol{R}(n)$, conjectures proposed like this:

\section{CONJECTURE 1}

1) $\boldsymbol{R}\left(2^{n+1}\right)=\boldsymbol{Q}(n)=2^{2 n}$

2) $n / 2-1<\log _{2} \boldsymbol{R}(n)<n / 2+1$;

3) $\lim _{n \rightarrow \infty} \frac{\log _{2} R(n)}{n}=\frac{1}{2}$

Then, raise doubts on item 2). we shall list the related data as following for convenience.

Attention to this data:

WHEN $n=20$ and $\boldsymbol{R}(n)=2$ 203, GET $\log _{2} \boldsymbol{R}(n)=$ $11.10525, \boldsymbol{n} / \mathbf{2}=10.00000, \boldsymbol{n} / \mathbf{2}+1=11.00000$;

WHEN $n=37$ and $\boldsymbol{R}(n)=756839$, GET $\log _{2} \boldsymbol{R}(n)=$ $19.52963, n / 2=18.50000, n / 2+1=19.50000$;

WHEN $n=41, \boldsymbol{R}(n)=2976$ 221, GET $\log _{2} \boldsymbol{R}(n)=$ $21.50505, n / 2=20.50000, n / 2+1=21.50000$;

WHEN $n=43, \boldsymbol{R}(n)=6972$ 593, GET $\log _{2} \boldsymbol{R}(n)=$ $22.73326, \boldsymbol{n} / \mathbf{2}=21.50000, \boldsymbol{n} / \mathbf{2}+1=22.50000$;

WHEN $n=44, \boldsymbol{R}(n)=13466917$, GET $\log _{2} \boldsymbol{R}(n)=$ $23.68292, \boldsymbol{n} / \mathbf{2}=22.00000, \boldsymbol{n} / \mathbf{2}+1=23.00000$

The location of Mersenne primes ignored for they have not been settled when $20996011 \leq p \leq 43112609$. The data listed above satisfied the formula $\log _{2} \boldsymbol{R}(n)>$ $\mathbf{n} / \mathbf{2}+\mathbf{1}$, and item 2) in the conjecture follows the formula $\log _{2} \boldsymbol{R}(n)<\boldsymbol{n} / \mathbf{2}+1$ which contradicts the result $\log _{2} \boldsymbol{R}(n)>$ $\boldsymbol{n} / \mathbf{2}+1$, so it concludes that the formula $\log _{2} \boldsymbol{R}(n)<\boldsymbol{n} / \mathbf{2}+$ 1 in conjecture $\boldsymbol{n} / \mathbf{2}-1<\log _{2} \boldsymbol{R}(n)<\boldsymbol{n} / \mathbf{2}+1$ is wrong.

In Chen's paper, the author presupposed that $2^{\frac{n+3}{2}}<$ $\boldsymbol{P}(n)<2^{\frac{n+7}{2}}$, while $\boldsymbol{P}(31) \leq \boldsymbol{P}(n) \leq \boldsymbol{P}(58)$ according to conjecture $\boldsymbol{n} / \mathbf{2}-1<\log _{2} \boldsymbol{R}(n)<\boldsymbol{n} / \mathbf{2}+1$. According to Chen's deduction, a great difference occurred on some numbers. It will be subscribed by the location-settled Mersenne primes as following.

WHEN $\boldsymbol{R}(32)=756839,2^{19.5}<741456$, GET 756
$839<741$ 456, impossible;

WHEN $\boldsymbol{R}(36)=2976221,2^{21.5}<2965821$, GET 2 $976221<2965821$, impossible;

WHEN $\boldsymbol{R}(38)=6972593,2^{22.5}<5931642$, GET 6 $972593<5931642$, impossible;

\subsection{Different Views}

Scholars proposed many conjectures on how to confirm the quantity of Mersenne primes in certain range. In 1980, Lenstra and Pomerance [6] independently presupposed the quantity of Mersenne primes while less than $x$, which would be $\left(e^{\gamma} / \log 2\right) \log \log x$, as $\gamma=0.5772$ is the Euler's constant. Based on that, Wagstaff presupposed a conjecture in 1983, as following:

CONJECTURE 2 [7]

1) IF the quantity of Mersenne primes which less than $x$ is $\pi_{M}(x)$, Then

$$
\pi_{M}(x) \approx \frac{e^{\gamma}}{\ln 2} \log \log x=(2.5695 \cdots) \ln \ln x,
$$

As $\gamma$ is the Euler's constant;

2) the expect value of Mersenne primes $M_{q}$ is about $e^{\gamma}=1.7806 \cdots$, while $x<q<2 x$;

3) the probability of $M_{q}$ is a prime number is about

$$
\frac{e^{\gamma}}{\ln 2} \cdot \frac{\ln a q}{\ln 2}=(2.5695 \cdots) \frac{\ln a q}{q},
$$

as $a=\left\{\begin{array}{ll}2 & q \equiv 3(\bmod 4) \\ 6 & q \equiv 1(\bmod 4)\end{array}\right.$.

This conjecture explains the probability of $M_{q}$ is a Mersenne prime in the precondition of $q$, and also pointed the quantity of Mersenne primes in certain range. It has been confirmed that there are 39 Mersenne primes when $p \leq 13466917$. However, the number

$$
\begin{aligned}
& \pi_{M}(x) \approx \frac{e^{\gamma}}{\ln 2} \log \log 13466917, \\
& =(2.5695 \cdots) \ln \ln 13466917 \approx 7.190360
\end{aligned}
$$

quite differs from the actual situation which could be thought that the conjecture 1) is not advisable. Based on conjecture 3), take $q=521$ and $q=257$ in Mersenne conjecture as an example, for $521 * 2571(\bmod 4)$, while $a=6$, then we get

$$
\begin{aligned}
& (2.5695 \cdots) \frac{\ln a q}{q}=(2.5695 \cdots) \frac{\ln (6 \cdot 521)}{521} \approx 0.039691 \\
& (2.5695 \cdots) \frac{\ln a q}{q}=(2.5695 \cdots) \frac{\ln (6 \cdot 257)}{257} \approx 0.073397
\end{aligned}
$$

It's well known that $M_{521}$ is a Mersenne prime, but $M_{257}$. It could meet a big mistake to some extent if we 
Table 1. Relationship between $R(n)$ and $n$ [5].

\begin{tabular}{|c|c|c|c|c|c|c|c|c|c|}
\hline$n$ & $R(n)$ & $P, Q$ & $\log _{2} R(n)$ & $n / 2$ & $n$ & $R(n)$ & $P, Q$ & $\log _{2} R(n)$ & $n / 2$ \\
\hline 1 & 2 & $\boldsymbol{P}(1)$ & 1.00000 & 0.50000 & 27 & 11213 & $\boldsymbol{P}(23)$ & 13.45288 & 13.50000 \\
\hline 2 & 2 & $\boldsymbol{Q}(0)$ & 1.00000 & 1.00000 & 28 & 19937 & $\boldsymbol{P}(24)$ & 14.28316 & 14.00000 \\
\hline 3 & 3 & $\boldsymbol{P}(2)$ & 1.58496 & 1.50000 & 29 & 21701 & $\boldsymbol{P}(25)$ & 14.40547 & 14.50000 \\
\hline 4 & 4 & $Q(1)$ & 2.00000 & 2.00000 & 30 & 23209 & $\boldsymbol{P}(26)$ & 14.50240 & 15.00000 \\
\hline 5 & 5 & $\boldsymbol{P}(3)$ & 2.32193 & 2.50000 & 31 & 44497 & $\boldsymbol{P}(27)$ & 15.44142 & 15.50000 \\
\hline 6 & 7 & $\boldsymbol{P}(4)$ & 2.80735 & 3.00000 & 32 & 65536 & $Q(4)$ & 16.00000 & 16.00000 \\
\hline 7 & 13 & $\boldsymbol{P}(5)$ & 3.70044 & 3.50000 & 33 & 86243 & $\boldsymbol{P}(28)$ & 16.39612 & 16.50000 \\
\hline 8 & 16 & $\boldsymbol{Q}(2)$ & 4.00000 & 4.00000 & 34 & 110503 & $\boldsymbol{P}(29)$ & 16.75373 & 17.00000 \\
\hline 9 & 17 & $\boldsymbol{P}(6)$ & 4.08746 & 4.50000 & 35 & 132049 & $\boldsymbol{P}(30)$ & 17.01071 & 17.50000 \\
\hline 10 & 19 & $\boldsymbol{P}(7)$ & 4.24793 & 5.00000 & 36 & 216091 & $\boldsymbol{P}(31)$ & 17.72128 & 18.00000 \\
\hline 11 & 31 & $\boldsymbol{P}(8)$ & 4.95420 & 5.50000 & 37 & 756839 & $\boldsymbol{P}(32)$ & 19.52963 & 18.50000 \\
\hline 12 & 61 & $\boldsymbol{P}(9)$ & 5.93074 & 6.00000 & 38 & 859433 & $\boldsymbol{P}(33)$ & 19.71303 & 19.00000 \\
\hline 13 & 89 & $\boldsymbol{P}(10)$ & 6.47573 & 6.50000 & 39 & 1257787 & $\boldsymbol{P}(34)$ & 20.26246 & 19.50000 \\
\hline 14 & 107 & $\boldsymbol{P}(11)$ & 6.74147 & 7.00000 & 40 & 1398269 & $\boldsymbol{P}(35)$ & 20.41521 & 20.00000 \\
\hline 15 & 127 & $\boldsymbol{P}(12)$ & 6.89968 & 7.50000 & 41 & 2976221 & $\boldsymbol{P}(36)$ & 21.50505 & 20.50000 \\
\hline 16 & 256 & $Q(3)$ & 8.00000 & 8.00000 & 42 & 3021377 & $\boldsymbol{P}(37)$ & 21.52677 & 21.00000 \\
\hline 17 & 521 & $\boldsymbol{P}(13)$ & 9.02514 & 8.50000 & 43 & 6972593 & $\boldsymbol{P}(38)$ & 22.73326 & 21.50000 \\
\hline 18 & 607 & $\boldsymbol{P}(14)$ & 9.24555 & 9.00000 & 44 & 13466917 & $\boldsymbol{P}(39)$ & 23.68292 & 22.00000 \\
\hline 19 & 1279 & $\boldsymbol{P}(15)$ & 10.32080 & 9.50000 & 45 & 20996011 & $P(?)$ & 24.32361 & 22.50000 \\
\hline 20 & 2203 & $\boldsymbol{P}(16)$ & 11.10525 & 10.00000 & 46 & 24036583 & $P(?)$ & 24.51873 & 23.00000 \\
\hline 21 & 2281 & $\boldsymbol{P}(17)$ & 11.15545 & 10.50000 & 47 & 25964951 & $P(?)$ & 24.63006 & 23.50000 \\
\hline 22 & 3217 & $\boldsymbol{P}(18)$ & 11.65150 & 11.00000 & 48 & 30402457 & $P(?)$ & 24.85768 & 24.00000 \\
\hline 23 & 4253 & $\boldsymbol{P}(19)$ & 12.05427 & 11.50000 & 49 & 32582657 & $P(?)$ & 24.95760 & 24.50000 \\
\hline 24 & 4423 & $\boldsymbol{P}(20)$ & 12.11081 & 12.00000 & 50 & 37156667 & $P(?)$ & 25.14712 & 25.00000 \\
\hline 25 & 9689 & $\boldsymbol{P}(21)$ & 13.24213 & 12.50000 & 50 & 42643801 & $P(?)$ & 25.34583 & 25.50000 \\
\hline 26 & 9941 & $\boldsymbol{P}(22)$ & 13.27918 & 13.00000 & 52 & 43112609 & $P(?)$ & 25.36161 & 26.00000 \\
\hline
\end{tabular}

Note: The symbol ? means the location of those Mersenne primes haven't been settled.

judge from higher probability.

\section{Conclusions}

Starting from analyzing of the known Mersenne primes, different ideas proposed about the distributional conjectures of Mersenne primes, which would be beneficial to the studies on the quantity of Mersenne primes and the distribution of its index prime $p$.

\section{References}

[1] Q. Chen and P. Zhang, "A Math Treasure: Mersenne Primes," Encyclopedic Knowledge, Vol. 32, No. 5, 2009, p. 22.

[2] K. H. Rosen, "Elementary Number Theory and it's Ap- 
plications," Published by arrangement with the original publisher, Pearson Education, Inc., publishing as Addision-Wesley, 2005.

[3] H. Z. Zhou, "The Distribution of Mersenne Primes," Acta Scientiarum Naturalium Universitatis Sunyatseni, Vol. 31, No. 4, 1992, pp. 121-122.

[4] J. Z. Zhang, "Zhou Conjecture Reveals the Beauty of Mathematics," In: J. Z. Zhang, et al., Ed., 100 Cases of Scientific and Technological Achievements in Three Decades: 1978-2008, Children's Press, Wuhan: Hubei, 2008, pp. 8-9.
[5] S. W. Chen, "Conjecture on Distribution of Mersenne Primes," Huanghuai Journal, Vol. 11, No. 4, 1995, pp. 44-46.

[6] C. Pomerance, "Recent Developments in Primality Testing," Mathematical Intelligencer, Vol. 3, No. 3, 1980, pp. 97-105.

[7] S. Wagstaff, "Divisors of Mersenne Numbers," Mathematics and Computation, Vol. 40, No. 38, 1983, pp. 385 -397 . 